Supplementary Materials for "An Ab Initio and Density Functional Theory Study of the Structure and Bonding of Sulfur Ylides,” J. M. Standard, B. A. Copack, T. K. Johnson, D. E. Przybyla, S. R. Graham, and R. J. Steidl

Cartesian coordinates $(\AA)$, electronic energies ( $\mathrm{E}_{\mathrm{el}}$, a.u.), and vibrational zero-point energies (VZPE, a.u.) of sulfur ylides. The atom-numbering scheme is shown in Figure 1 of the manuscript.

1. $\mathrm{H}_{2} \mathrm{~S}-\mathrm{CH}_{2}, \mathrm{~B} 3 \mathrm{LYP} / \mathrm{aug}-\mathrm{cc}-\mathrm{pV}(\mathrm{D}+\mathrm{d}) \mathrm{Z}$

$\mathrm{E}_{\mathrm{el}}=-438.6286891 ; \mathrm{VZPE}=0.041567$

$\begin{array}{rrrr}\mathrm{C} & -1.155183 & 0.061952 & -0.045689 \\ \mathrm{H} & -1.656984 & -0.897746 & 0.055816 \\ \mathrm{H} & -1.628169 & 0.963863 & 0.336552 \\ \mathrm{~S} & 0.500184 & -0.107649 & -0.031442 \\ \mathrm{H} & 1.187527 & 0.340118 & 1.116799 \\ \mathrm{H} & 1.025774 & 0.944439 & -0.731965\end{array}$

2. $\mathrm{H}_{2} \mathrm{~S}-\mathrm{CH}_{2}, \mathrm{~B} 3 \mathrm{LYP} / \mathrm{aug}-\mathrm{cc}-\mathrm{pV}(\mathrm{T}+\mathrm{d}) \mathrm{Z}$

$\mathrm{E}_{\mathrm{el}}=-438.6593098 ; \mathrm{VZPE}=0.041863$

$\begin{array}{rrrr}\mathrm{C} & -1.141858 & 0.059337 & -0.035505 \\ \mathrm{H} & -1.656739 & -0.882789 & 0.042638 \\ \mathrm{H} & -1.624916 & 0.977596 & 0.253014 \\ \mathrm{~S} & 0.495660 & -0.108843 & -0.022947 \\ \mathrm{H} & 1.158118 & 0.421664 & 1.082613 \\ \mathrm{H} & 1.044123 & 0.868995 & -0.798079\end{array}$

3. $\mathrm{H}_{2} \mathrm{~S}-\mathrm{CH}_{2}, \mathrm{~B} 3 \mathrm{LYP} /$ aug-cc-pV(Q+d)Z

$E_{\mathrm{el}}=-438.6661098 ; \mathrm{VZPE}=0.041852$

$\begin{array}{rrrr}\mathrm{C} & -1.140062 & 0.059009 & -0.033263 \\ \mathrm{H} & -1.657240 & -0.881564 & 0.039689 \\ \mathrm{H} & -1.625711 & 0.980656 & 0.237142 \\ \mathrm{~S} & 0.495025 & -0.109076 & -0.021494 \\ \mathrm{H} & 1.154630 & 0.436349 & 1.076580 \\ \mathrm{H} & 1.048297 & 0.855715 & -0.809926\end{array}$

4. $\mathrm{H}_{2} \mathrm{~S}-\mathrm{CH}_{2}, \mathrm{MP} 2 /$ aug-cc-pV(D+d)Z

$\mathrm{E}_{\mathrm{el}}=-437.9913739 ; \mathrm{VZPE}=0.042871$

$\begin{array}{rrrr}\mathrm{C} & -1.158929 & 0.062596 & -0.056564 \\ \mathrm{H} & -1.642432 & -0.906306 & 0.067874 \\ \mathrm{H} & -1.622376 & 0.951173 & 0.372027 \\ \mathrm{~S} & 0.501709 & -0.105044 & -0.030704 \\ \mathrm{H} & 1.172010 & 0.319541 & 1.111264 \\ \mathrm{H} & 1.019017 & 0.940710 & -0.720521\end{array}$


5. $\mathrm{H}_{2} \mathrm{~S}-\mathrm{CH}_{2}, \mathrm{MP} 2 /$ aug-cc-pV(T+d)Z

$\begin{array}{lrrr}\mathrm{E}_{\mathrm{el}}=-438.1510378 ; \mathrm{VZPE}=0.043165 & \\ \mathrm{H} & -1.136934 & 0.058138 & -0.039085 \\ \mathrm{C} & -1.639036 & -0.884734 & 0.048754 \\ \mathrm{H} & -1.615951 & 0.972903 & 0.249735 \\ \mathrm{H} & 0.493973 & -0.106958 & -0.020464 \\ \mathrm{~S} & 1.135945 & 0.428900 & 1.065809 \\ \mathrm{H} & 1.037082 & 0.845440 & -0.802360 \\ \mathrm{H} & & \end{array}$

\section{6. $\mathrm{H}_{2} \mathrm{~S}-\mathrm{CH}_{2}, \mathrm{MP} 2 /$ aug-cc-pV(Q+d)Z}

$\mathrm{E}_{\mathrm{el}}=-438.2118984 ; \mathrm{VZPE}=0.043019$

$\begin{array}{rrrr}\mathrm{C} & -1.132971 & 0.057023 & -0.029794 \\ \mathrm{H} & -1.643047 & -0.883539 & 0.035297 \\ \mathrm{H} & -1.619360 & 0.986384 & 0.193615 \\ \mathrm{~S} & 0.492312 & -0.107551 & -0.015885 \\ \mathrm{H} & 1.129437 & 0.475294 & 1.046159 \\ \mathrm{H} & 1.053801 & 0.800536 & -0.842145\end{array}$

7. $\left(\mathrm{CH}_{3}\right)_{2} \mathrm{~S}-\mathrm{CH}_{2}, \mathrm{~B} 3 \mathrm{LYP} /$ aug-cc-pV(D+d)Z

$\begin{array}{lrrr}\mathrm{E}_{\mathrm{el}}=-517.2759990 ; \mathrm{VZPE}=0.099172 & \\ \mathrm{C} & -1.633166 & -0.224868 & 0.316181 \\ \mathrm{C} & -2.438859 & -0.581820 & -0.317309 \\ \mathrm{H} & -1.708136 & -0.260704 & 1.401713 \\ \mathrm{H} & -0.179973 & -0.042403 & -0.446462 \\ \mathrm{~S} & 1.128452 & -1.192358 & 0.202110 \\ \mathrm{C} & 0.615766 & 1.488593 & 0.181200 \\ \mathrm{C} & 1.189031 & -1.100722 & 1.294372 \\ \mathrm{H} & 2.099805 & -0.965137 & -0.258225 \\ \mathrm{H} & 0.805534 & -2.202754 & -0.071011 \\ \mathrm{H} & 0.636843 & 1.464714 & 1.278272 \\ \mathrm{H} & -0.005146 & 2.322538 & -0.161483 \\ \mathrm{H} & 1.634188 & 1.574137 & -0.219877 \\ \mathrm{H} & & \end{array}$

8. $\left(\mathrm{CH}_{3}\right)_{2} \mathrm{~S}-\mathrm{CH}_{2}, \mathrm{~B} 3 \mathrm{LYP} /$ aug-cc-pV(T+d)Z

$$
\begin{array}{lrrr}
\mathrm{E}_{\mathrm{el}}=-517.3324114 ; \mathrm{VZPE}=0.099923 & \\
\mathrm{C} & -1.627237 & -0.210202 & 0.313996 \\
\mathrm{H} & -2.434150 & -0.543636 & -0.314876 \\
\mathrm{H} & -1.707503 & -0.242992 & 1.390194 \\
\mathrm{~S} & -0.181133 & -0.039794 & -0.442352 \\
\mathrm{C} & 1.110407 & -1.199872 & 0.199558 \\
\mathrm{C} & 0.631290 & 1.476766 & 0.180005 \\
\mathrm{H} & 1.160829 & -1.124956 & 1.284881 \\
\mathrm{H} & 2.079189 & -0.970995 & -0.244135 \\
\mathrm{H} & 0.790527 & -2.197518 & -0.088801 \\
\mathrm{H} & 0.652938 & 1.456032 & 1.268549 \\
\mathrm{H} & 0.026866 & 2.312044 & -0.161578 \\
\mathrm{H} & 1.642671 & 1.548578 & -0.217960
\end{array}
$$

9. $\left(\mathrm{CH}_{3}\right)_{2} \mathrm{~S}-\mathrm{CH}_{2}, \mathrm{~B} 3 \mathrm{LYP} /$ aug-cc-pV(Q+d)Z

$\mathrm{E}_{\mathrm{el}}=-517.3450182 ; \mathrm{VZPE}=0.099943$ 


$\begin{array}{lrrr}\mathrm{C} & -1.628435 & -0.181657 & 0.314021 \\ \mathrm{H} & -2.448652 & -0.471215 & -0.318302 \\ \mathrm{H} & -1.712481 & -0.210318 & 1.389463 \\ \mathrm{~S} & -0.181870 & -0.034336 & -0.441796 \\ \mathrm{C} & 1.079792 & -1.222517 & 0.197865 \\ \mathrm{C} & 0.665588 & 1.461870 & 0.180975 \\ \mathrm{H} & 1.130303 & -1.151262 & 1.283017 \\ \mathrm{H} & 2.053991 & -1.015039 & -0.243188 \\ \mathrm{H} & 0.738789 & -2.211995 & -0.092354 \\ \mathrm{H} & 0.691995 & 1.437848 & 1.268919 \\ \mathrm{H} & 0.078207 & 2.310846 & -0.154947 \\ \mathrm{H} & 1.676108 & 1.514328 & -0.221032\end{array}$

10. $\left(\mathrm{CH}_{3}\right)_{2} \mathrm{~S}-\mathrm{CH}_{2}$, MP2/aug-cc-pV(D+d)Z

$\mathrm{E}_{\mathrm{el}}=-516.3884316 ; \mathrm{VZPE}=0.100855$

$\begin{array}{lrrr}\mathrm{C} & -1.614076 & -0.311815 & 0.316294 \\ \mathrm{H} & -2.334689 & -0.873151 & -0.276762 \\ \mathrm{H} & -1.640032 & -0.362675 & 1.407982 \\ \mathrm{~S} & -0.167301 & -0.064218 & -0.458108 \\ \mathrm{C} & 1.217032 & -1.079833 & 0.214984 \\ \mathrm{C} & 0.476890 & 1.506282 & 0.178432 \\ \mathrm{H} & 1.256603 & -0.948869 & 1.307527 \\ \mathrm{H} & 2.173455 & -0.788868 & -0.247805 \\ \mathrm{H} & 0.977716 & -2.123673 & -0.028817 \\ \mathrm{H} & 0.454117 & 1.475866 & 1.278194 \\ \mathrm{H} & -0.193421 & 2.294179 & -0.187028 \\ \mathrm{H} & 1.503989 & 1.666879 & -0.181823\end{array}$

11. $\left(\mathrm{CH}_{3}\right)_{2} \mathrm{~S}-\mathrm{CH}_{2}, \mathrm{MP} 2 /$ aug-cc-pV(T+d)Z

$\mathrm{E}_{\mathrm{el}}=-516.6595256 ; \mathrm{VZPE}=0.101686$

$\begin{array}{lrrr}\mathrm{C} & -1.607601 & -0.184123 & 0.316886 \\ \mathrm{H} & -2.419085 & -0.518791 & -0.298526 \\ \mathrm{H} & -1.662147 & -0.209866 & 1.391334 \\ \mathrm{~S} & -0.173826 & -0.038681 & -0.451569 \\ \mathrm{C} & 1.075079 & -1.183362 & 0.204583 \\ \mathrm{C} & 0.637207 & 1.436421 & 0.181976 \\ \mathrm{H} & 1.104794 & -1.080140 & 1.286278 \\ \mathrm{H} & 2.049743 & -0.974261 & -0.229665 \\ \mathrm{H} & 0.744105 & -2.180534 & -0.063866 \\ \mathrm{H} & 0.624401 & 1.393742 & 1.267830 \\ \mathrm{H} & 0.053632 & 2.282512 & -0.161913 \\ \mathrm{H} & 1.657665 & 1.492623 & -0.187040\end{array}$


12. $\left(\mathrm{CH}_{3}\right)_{2} \mathrm{~S}-\mathrm{CH}_{2}, \mathrm{MP} 2 /$ aug-cc-pV(Q+d)Z

$\mathrm{E}_{\mathrm{el}}=-516.7632774$ (no VZPE was calculated for this case)

$\begin{array}{lrrr}\mathrm{C} & -1.612515 & -0.127601 & 0.316275 \\ \mathrm{H} & -2.449081 & -0.361230 & -0.312352 \\ \mathrm{H} & -1.678640 & -0.143031 & 1.390734 \\ \mathrm{~S} & -0.177170 & -0.027118 & -0.447536 \\ \mathrm{C} & 1.014087 & -1.231359 & 0.199116 \\ \mathrm{C} & 0.710360 & 1.406994 & 0.182955 \\ \mathrm{H} & 1.045576 & -1.145296 & 1.281444 \\ \mathrm{H} & 1.998662 & -1.059967 & -0.226545 \\ \mathrm{H} & 0.643680 & -2.209590 & -0.082325 \\ \mathrm{H} & 0.717242 & 1.361640 & 1.268030 \\ \mathrm{H} & 0.160730 & 2.280329 & -0.145225 \\ \mathrm{H} & 1.724960 & 1.422832 & -0.203253\end{array}$

